\title{
Study of Delays to Evaluate the Sustainability over Time of Solutions for Urban Intersections
}

\author{
F. Medina ${ }^{1}$, A. Burga ${ }^{1}$, A. Bravo ${ }^{2}$ \\ ${ }^{1}$ Research Student. Faculty of Civil Engineering, UPC, Lima, Perú \\ u201420103@upc.edu.pe; u201519041@upc.edu.pe \\ ${ }^{2}$ Full Professor. Faculty of Civil Engineering, UPC, Lima, Perú \\ aldo.bravo@upc.pe
}

\begin{abstract}
A way to evaluate if the solutions for urban intersections are sustainable in time, is about calculating the delays for a future traffic and the present study estimates the delay variation that are present in time to get a more accurate analysis when it is pretended to use in future traffic. The vehicular delay is one of the most important parameters that is used to evaluate the performance of traffic light intersections, for this reason, professionals of transport use as traffic optimization judgment the decrease of delays This article presents an approach to evaluate the variation of delays through the time for urban intersections for which this investigation analyses the delays in one intersection for different stages. This project begins with a model of the intersection of study helped by the software Synchro 10. Then, the model will be applied for many stages where the vehicular volume will be increased depending the vehicular growth. Finally, it is proved that due to the estimated delays for each stage, it will be possible to estimate their own variations that is based on the trend of percentages variation curve on time delays. In brief, the methodology could be applied in different urban intersections and stages where it is pretended to propose solutions that are sustainable over the time.
\end{abstract}

Keywords: Future traffic; Delays; Traffic light intersection; Vehicular congestion.

\section{Introduction}

A method for evaluating if the proposed solutions to improve the performance of intersections are sustainable in time, it is about estimating the delay for a future traffic and this investigation will estimate the different variations that delays introduce in time. The growth of the automotive market in the main cities of the world has been increased [1]. This increase is closely related to the improvement of road infrastructure, the growth of the population in general and the economic takeoff of the countries, generating a significant increase in traffic congestion. In a city, the traffic congestion is characterized for many parameters; like the fast growth of population, the number of four wheels' vehicles, the unsuitable and deficient transport infrastructure and the lack of a master plan to govern the develop of improvement projects [2].

The increase of the automotive market in important cities aggravates the situation of ways each year. Due to the vial infrastructure cannot keeping the rhythm of vehicular growth, the situation generates an imbalance between the supply and demand [3]. For this reason, the investigation promotes two solution approaches, each one is focus on the requirement that the authors want. It is expected that these solutions are sustainable in the time that it is pretended to design. The traffic engineering with traffic predictions is a promising approach to settle that traffic that changes in time [4]. In addition, traffic prediction can reduce problems such as traffic congestions, accidents, environmental pollution, economic losses, and unnecessary waste of fuel, in metropolitan areas [5].

\section{Case of Study}

The study was accomplished at an intersection located in a consolidated urban area of the city of Lima. The Javier Prado Avenue is one of the principal ways of the Peruvian capital. The users of the intersection are pedestrians, cars, custers, buses and trucks. Little presence of motorcycles and bicycles. The intersection has three approaches and nine lanes, of which one lane has an exclusive left turn, the existing traffic light has a cycle of 180 seconds, three phases, one of them assigned to the exclusive turn, the service level (LOS) is F. This article presents the evaluation of future delays at the intersection of Avenida Javier Prado - Avenida San Luis, with the purpose of evaluating the sustainability of the solution over time. To improve the 
precision of the results, data was also collected from the streets, avenues, and passages within the environment of the study area.

\section{Methodology}

The methodology to apply in this study consists about estimating the delays of intersection before mentioned, for a future traffic until ten years with a linear growth of vehicle park, for which this project will use the Synchro 10 software. It is must necessary to get the information (vehicle capacity, geometry, traffic light cycles and pedestrian capacity) at the intersection to be studied. The linear growth of the vehicle delay at the intersection and the vehicle volume will be considerate as variables, where the delays will be the dependent variable. The process will be iterative, which will search the percentage variation of delays for future traffics.

\subsection{Data Collection and Analysis}

The prevailing conditions are determined by performing the following activities:

- A vehicle gauging (cars, buses, and trucks) of the intersection and its nearby streets is carried out for three days (two typical days and one atypical). Includes distribution by lanes and by movement.

- Information is collected on the geometry of the study area (lane widths, shoulder widths, lateral obstructions, characteristics of the alignments, and horizontal and vertical signs).

- The peak hour (one-hour time interval where the highest traffic density occurs) of the intersection is estimated.

- The pedestrian gauging is carried out at rush hour, for a single day.

- The traffic light cycle of the intersection is determined (phases, cycle length and green distributions), see Table 1.

Table 1: Current traffic light cycle in the intersection.

\begin{tabular}{|l|c|c|c|}
\cline { 2 - 4 } \multicolumn{1}{c|}{} & \multicolumn{3}{c|}{ Javier Prado Avenue \& San Luis Avenue } \\
\hline Time (s) & Green & Amber & Red \\
\hline E-W & 80 & 3 & 97 \\
\hline N-S / S-N & 63 & 3 & 114 \\
\hline S-N (Exclusive turn) & 25 & 3 & 152 \\
\hline
\end{tabular}

\subsection{Modeling in Synchro 10}

Synchro is a macroscopic analysis and optimization software application; it is compatible with Highway Capacity Manual's (HCM) 6th Edition. The objective of Synchro is about increasing the efficiency of traffic networks, among its functions one is to calculate the delay of traffic lights intersections [6]. The characteristics of the way and traffic were optimized.

Three scenarios were evaluated: current, optimizing traffic lights and increasing a lane. For the modeling and the obtaining of results the steps were the next following:

- The model of the case of study was created in the software Synchro according its geometrics parameters.

- Lane settings were entered, according the software and helped by the user guide [7].

- Input of volume Settings

- Estimate the delay at the intersection for the linear growth rate in Peru of $4.8 \%$ [8].

- Modifying of the vehicle growth factor, for each year until a future traffic of ten years. Consider linear rates from 3\% to $11 \%$.

\section{Results Discussion}




\subsection{Stage1: Current.}

The figure 1 shows a jump in the variation of delays for the intersection, this variation it is reduced when the percentage of vehicular growth decreases and shifts in time. Later, it could be seen that the trend of percentage variation in delays tends to normalize.

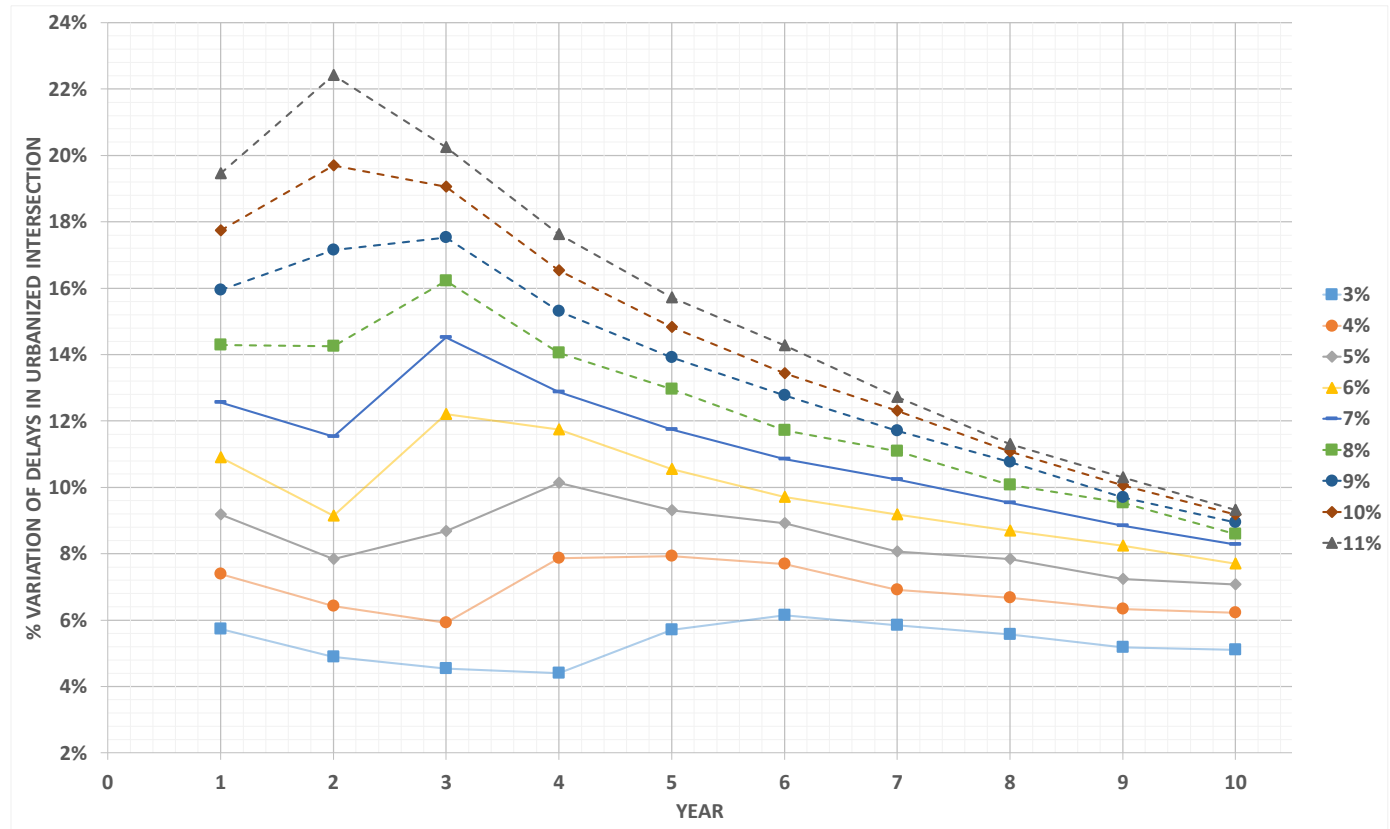

Fig.1: Percentage variation of delays from the last year, day one

\subsection{Stage 2: Optimizing traffic lights.}

In the analysis of the intersection for the day one including the traffic light optimization, the figure 2 shows in the graph a variation in the trend that the percentage curves changes of delays present. In comparison with the picture, this variation decreases by using alternative solutions for the intersection. In this case of study, the alternative solution was about optimizing the traffic light cycle. 


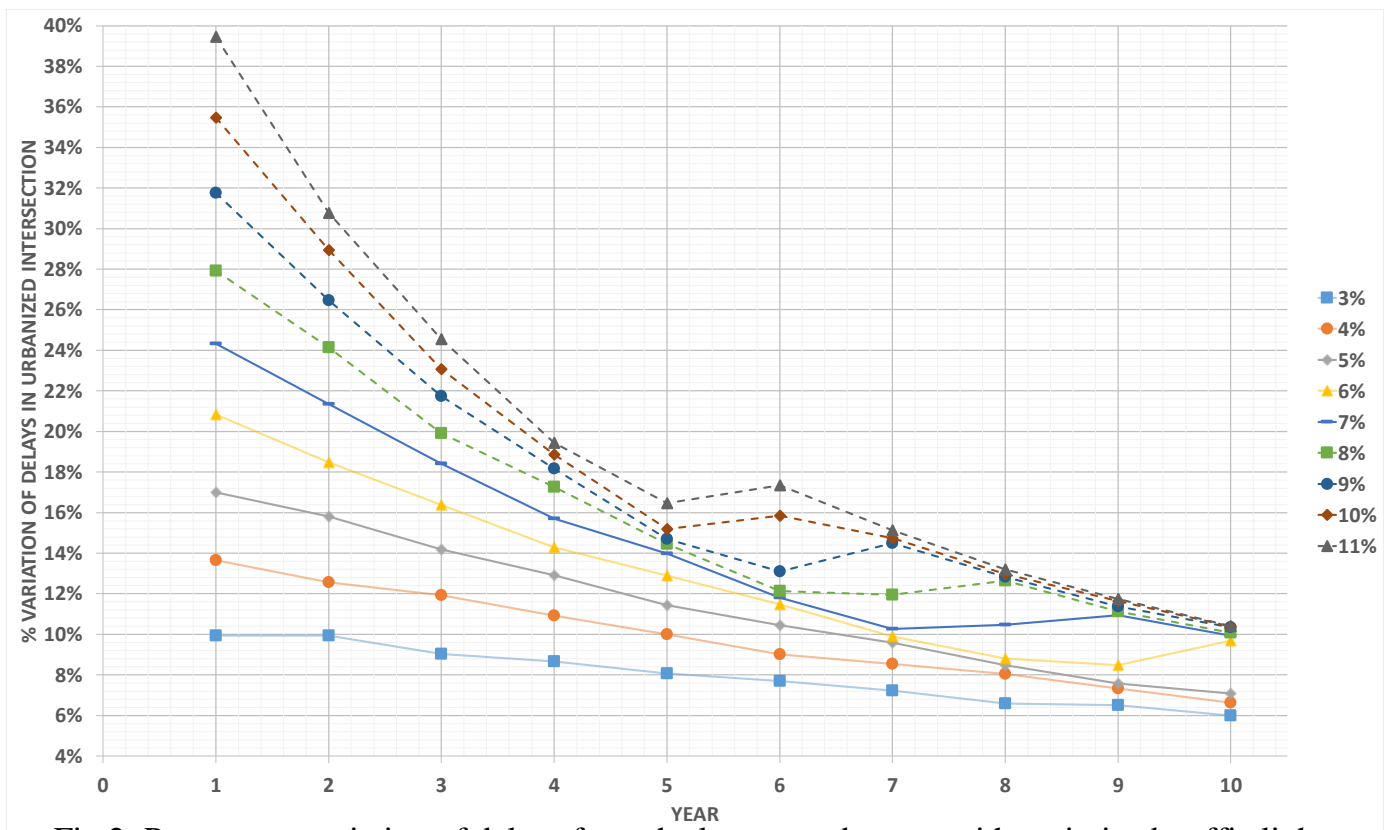

Fig.2: Percentage variation of delays from the last year, day one with optimized traffic light.

\subsection{Stage 3: Increasing A Lane and Optimizing Traffic Lights.}

The first delay in the intersection for the day two was 74 seconds. The figure 3 shows the curves with variation in a minor tendency comparing figure 1 and 2 . There is also a slight variation in the curves with a vehicle growth greater than $8 \%$ and for a future traffic of four years.

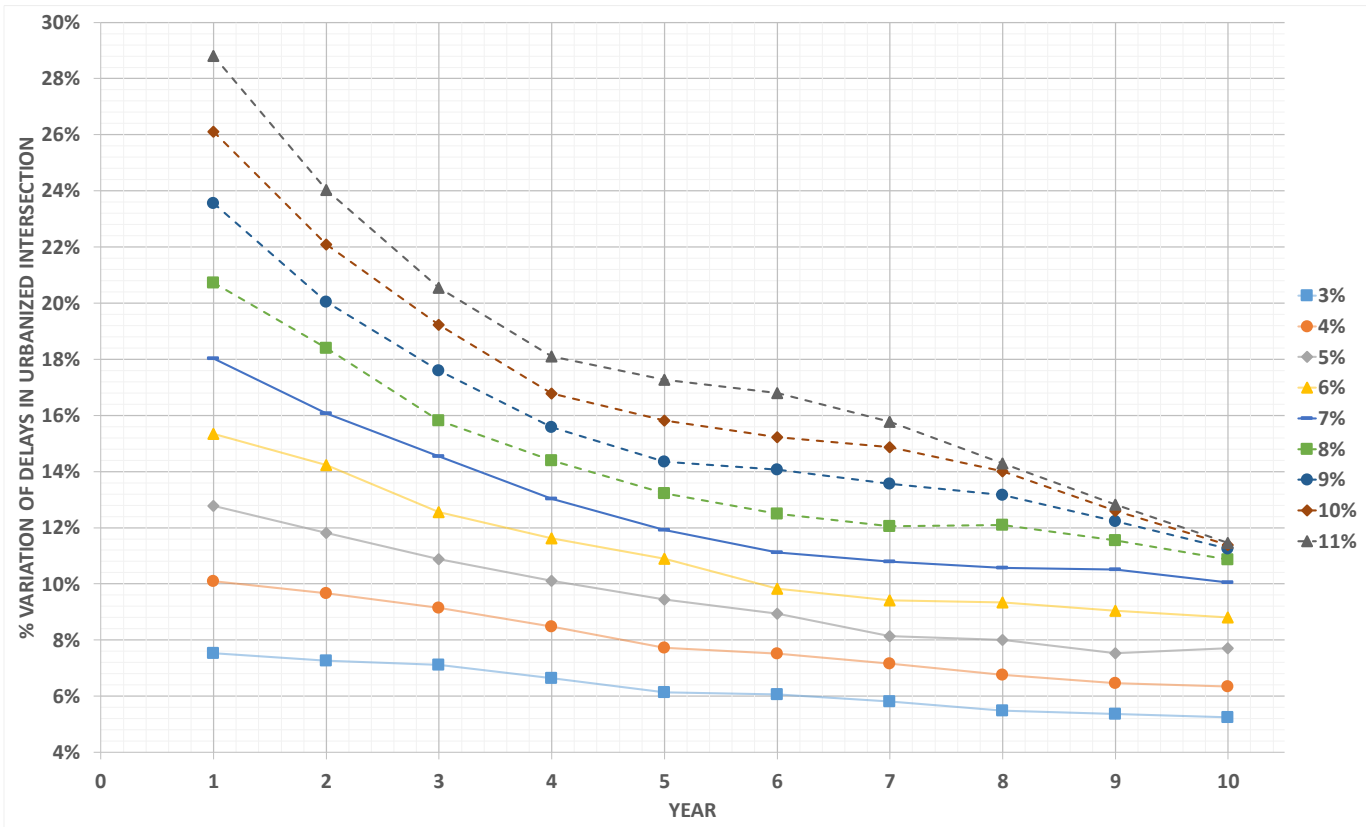

Fig. 3: Percentage variation of delays over the previous year, day one. 


\section{Conclusions}

- From this case of study, it is determined that the use of alternative solutions allow a properly function of a vehicular vehicular intersection. As a case of optimizing the traffic light cycle will allow the future traffic analysis to be more reliable reliable for its evaluation in intersections with a large traffic flow.

- If the optimization traffic lights are applied in cities where the annual vehicle growth is greater than $7 \%$ per year, the analysis for the estimation of future traffic is limited to five years. When the annual vehicle growth is less than 5\%, the future traffic calculation is suggested to be limited to ten years. And for values between 5\% and 7\% consider seven years maximum.

- If, in addition to the optimization of traffic lights, the number of lanes is increased, the estimate of future traffic is projected for ten years. This value coincides with the study periods developed by each urban district administration. This study is valid in consolidated urban areas where traffic growth usually tends to decrease, and the traffic attracted is low or null.

\section{References}

[1] OIT, Issues paper for the Technical Meeting on the Future of Work in the Automotive Industry (Geneva, 4-8 May 2020).

[2] A. Mohanty, S. Mahapatra, and U. Bhanja, 2019. Traffic congestion detection in a city using clustering techniques in VANETs vol. 13, no 3, pp. 884-891

[3] M. Errampalli, R. Kayitha 2016. Traffic Management Plan for Port Blair City, India. Vol. 17, pp. 548-577

[4] T. Otoshi, Y. Ohsita, M. Murata, Y. Takahashi, K. Ishibashi, K. Shiomoto 2015. Traffic prediction for dynamic traffic engineering. Vol. 85, pp. 36-50

[5] S. Chavhan, P. Venkataram 2019. Prediction based traffic management in a metropolitan area

[6] Trafficware LLC, Synchro Studio 10 User Guide. Sugar Land, TX, 2017

[7] K. Udomsilp, T. Arayakarnkul, S. Watarakitpaisarn, P. Komolkiti, J. Rudjanakanoknad and C. Aswakul, 2017. Traffic Data Analysis on Sathorn Road with Synchro Optimization and Traffic Simulation. Vol. 11, no. 6

[8] Ministry of transport and communications. (2018). Statistical Yearbook 2017. Recovered from: http://portal.mtc.gob.pe/estadisticas/publicaciones/anuarios/ANUARIO_ESTADISTICO_2017.pdf Accessed: March 13, 2019. 